\title{
Novel COL9A3 mutation in a family diagnosed with multiple epiphyseal dysplasia: a case report
}

\author{
Changhoon Jeong ${ }^{1}$, Jae Young Lee ${ }^{1}$, Jiyeon Kim², Hyojin Chae ${ }^{2,3}$, Hae-il Park ${ }^{3}$, Myungshin Kim, ${ }^{2,{ }^{*}}$, Ok-Hwa Kim ${ }^{4}$
} Paul Kim ${ }^{5}$, Young Kee Lee ${ }^{5}$ and Jongsun Jung ${ }^{5}$

\begin{abstract}
Background: Multiple epiphyseal dysplasia is a common skeletal dysplasia characterized by mild short stature, early-onset osteoarthritis mainly involving the hip and knee joints, and abnormally small and/or irregular epiphyses. Multiple epiphyseal dysplasia is clinically and genetically heterogeneous and six genes are associated with the phenotype of multiple epiphyseal dysplasia.

Case presentation: A 12-year-old Korean boy presented with intermittent knee pain. His height was $144.6 \mathrm{~cm}$ (20th percentile) and family history was notable for early-onset osteoarthritis in his father. The proband's x-rays revealed epiphyseal changes characteristic of multiple epiphyseal dysplasia associated with a collagen IX defect, with manifestations primarily restricted to the knees. Mutational analysis identified a novel c.104G > A substitution in exon 2 of COL9A3, resulting in p.Gly35Asp in the proband and his father. In silico analyses predicted the p.Gly35Asp amino acid change to be detelerious, and molecular dynamics simulation demonstrated a major structural change in the heterotrimeric collagen IX.
\end{abstract}

Conclusion: So far, three COLAA3 mutations, have been reported. These three mutations are located at the splice donor or acceptor site of COL9A3 and cause skipping of exon 3, resulting in the deletion of 12 aminoacids in the COL3 domain of COL9A3. In contrast, the novel missense mutation identified in this two-generation family with multiple epiphyseal dysplasia is a missense mutation affecting the Gly residue of the Pro-Pro-Gly repeat sequence in the COL3 domain of collage IX, with accompanying major structural change of the collagen peptide.

Keywords: Multiple epiphyseal dysplasia, COL9A3, Molecular dynamics simulation

\section{Background}

Multiple epiphyseal dysplasia (MED) is clinically and genetically heterogeneous group of skeletal dysplasias characterized by early-onset osteoarthritis, a waddling gait, restriction of joint mobility, pain and stiffness in the weight-bearing joints and sometimes short stature. Mutations in six different genes cause MED. Mutations in genes encoding cartilage oligomeric matrix protein (COMP), matrilin-3 (MATN3), and the alpha 1-3 chains of type IX collagen (COL9A1, COL9A2, COL9A3) result in autosomal dominant MED [1], and a specific mutation in the diastrophic dysplasia sulfate transporter $(D T D S T)$ is

\footnotetext{
* Correspondence: microkim@catholic.ac.kr

${ }^{2}$ Catholic Genetic Laboratory Center, Seoul St. Mary' Hospital, The Catholic

University of Korea, Seoul, Korea

${ }^{3}$ Department of Laboratory Medicine, The Catholic University of Korea, Seoul, Korea

Full list of author information is available at the end of the article
}

associated with an autosomal recessive form of MED [2]. The frequencies of mutations in these genes among MED patients have yet to be determined. Previous studies in European MED patients have shown frequencies of 7-35\% for COMP, $14 \%$ for DTDST, 5-10\% for MATN3, and $5-15 \%$ for the type IX collagen genes $[3,4]$. The frequencies of causative genes showed racial differences. A comprehensive screening in a Japanese population indicated that frequencies differ in East Asian patients, with reported frequencies of $23 \%$ in MATN3, 20\% in COMP, $6 \%$ in COL9A2, and none in DTDST [5]. In Korean patients, MATN3 mutations were found in $55 \%$ of patients, followed by COMP mutations in $41 \%$, and COL9A2 and DTDST mutations in one patients (2\%), respectively [6]. Genotype-phenotype correlations of MED has been described and MED resulting from collagen IX defects (COL9-MED) result in more severe involvement of the knees with relative sparing of the hips. 
Here we report a two-generation family with MED phenotypes consistent with COL9-MED, that were caused by a novel missense mutation in COL9A3.

\section{Case presentation}

\section{Patient's characteristics}

The proband was a 12-year-old Korean boy born to nonconsanguineous parents. He had been treated for partial seizure disorder for 2 years. He was referred to the pediatric orthopedic clinic for the evaluation of intermittent knee pain that had occurred for a few months. His height was $144.6 \mathrm{~cm}$ (20th percentile) and bodyweight was $41 \mathrm{~kg}$ (60th percentile). The height of his father and mother was $170 \mathrm{~cm}$ and $160 \mathrm{~cm}$, respectively. His father also suffered from intermittent mild knee joint pain. The proband and his father did not show muscle weakness and pain suggesting myopathy. There was no Gower sign and Trendelenberg sign on physical examination. The level of creatine kinase and lactate were normal. Informed consent was obtained from the parents of the child.

\section{Orthopedic and radiologic evaluations}

Clinically, the lower extremity of the proband showed mild genu valgum deformity. The knee joints displayed full range of motion. The distal femoral and proximal tibial epiphyses were affected most, and the distal tibia and distal radius epiphyses were affected less severely. There was no irregularity on the femoral head epiphysis. Distal femoral and proximal tibial epiphyses showed loss of height, joint surface irregularities and fragmentation (Figure $1 \mathrm{~A}$ and $\mathrm{B}$ ). The epiphysis of the distal tibia showed lateral shortening and fragmentation (Figure $1 \mathrm{C}$ and D). There was no abnormality of the distal fibular epiphysis nor the talar dome. The tarsal navicular and $\mathrm{cu}-$ neiform bone showed irregular ossification (Figure 1E). The epiphysis of distal radius was wedged shape and the epiphyses of the distal ulnae were relatively small. The carpal bone shows dysplasia and flattening (Figure 1F). The spine and the proximal femoral epiphyses appeared normal (Figure 1G). X-rays of the proband's father revealed osteoarthritis of Kellgren-Lawrence grade I particularly on the medial side (Figure 2A). However, the proximal femoral epiphyses were spared (Figure 2B). Other members of the family were not affected.

\section{Genetic analyses}

PCR from genomic DNA followed by direct sequencing was performed following parental informed consent. Genomic DNA was isolated from peripheral blood leukocytes. Direct DNA sequencing of the COMP, MANT3, COL9A1, COL9A2, and COL9A3 genes was performed. All exons were amplified using the primers previously described (Table 1). PCR amplicons were bidirectionally sequenced with the Big Dye terminator v3.1 cycle sequencing kit (Applied Biosystems, Foster City, CA) using an ABI PRISM 3130 Genetic Analyzer (Applied Biosystems). COMP, MATN3, COL9A1 and COL9A2 revealed no mutations. A novel missense mutation c.104G $>A$ in exon2 of COL9A3, which resulted in p.Gly35Asp, was identified. RefSeq ID: NM_001853.3 reference sequence was used for cDNA nucleotide numbering. This mutation was predicted to be not tolerated by SIFT and probably

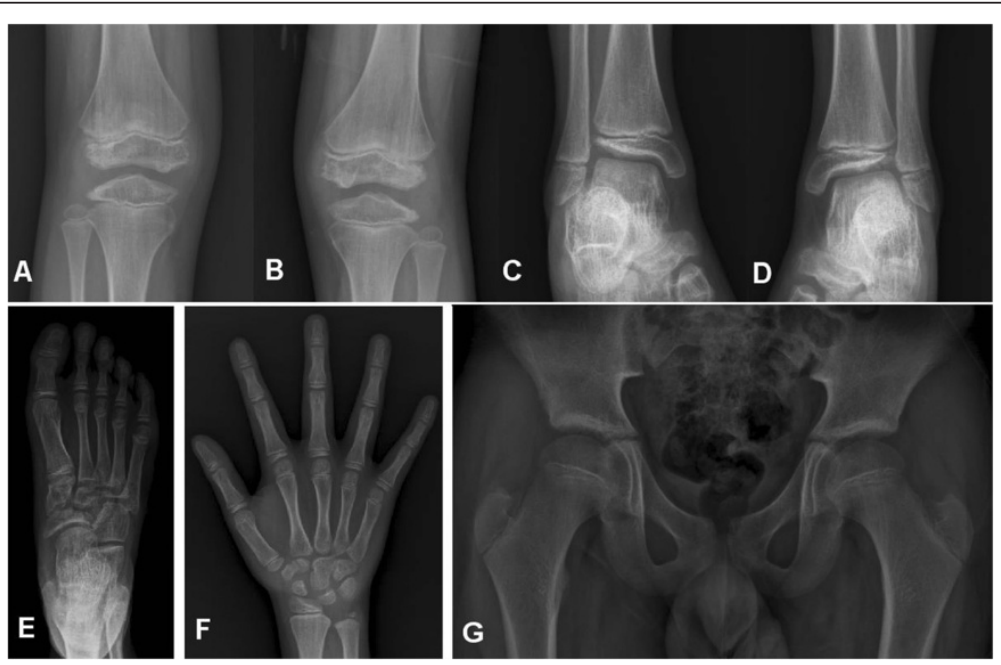

Figure 1 Radiographs of proband at the age of 12 years. Anteroposterior view of knee joint showed shortening, fragmentation, and joint surface irregularity of distal femoral and proximal tibial epiphysis (A, B). Anteroposterior view of distal tibial epiphysis showed a lateral wedging and fragmentation (C, D). The tarsal navicular and cuneiform bone showed irregular ossification (E). The epiphysis of distal radius was wedge shaped and the epiphyses of the distal ulnae were relatively small and the carpal bone shows dysplasia and flattening (F). The epiphyses of proximal femur were spared (G). 


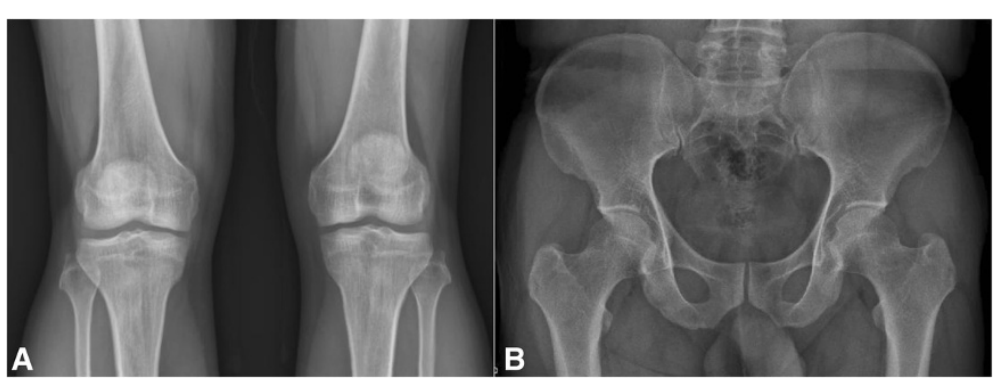

Figure $\mathbf{2}$ Radiographs of the proband's father at the age of $\mathbf{4 1}$ years. Anteroposterior view of knee joints revealed early osteoarthritis with findings including of medial joint space narrowing and joint surface irregularity (A). The capital femoral epiphysis of the proband's father has no evidence of dysplasia (B).

damaging by PolyPhen $[7,8]$. Also, comparative evolutionary analysis showed that the glycine residue at codon 35 is a highly conserved amino acid during evolution. And the same mutation was observed in the proband's symptomatic father. We performed Sanger sequencing of 100 healthy controls and none harbored a c.104G $>$ A mutation in exon 2 of COL9A3.

\section{Molecular Dynamics (MD) simulation of wild type and mutant model structure of trimer collagen IX}

Molecular mechanics potential energy minimization and MD simulations were carried out using the program package AMBER 11 [9]. FF99 force field in AMBER package was used in all MD simulations including hetero atoms. Topology file GAFF force field in AMBER package was used. The generalized Born solvation model was used instead of explicit water [10]. The temperature was kept constant by using Langevin dynamics with a collision frequency of $5.0 \mathrm{ps}^{-1}$ [11]. The minimized system was heated from $0 \mathrm{~K}$ to $325 \mathrm{~K}$ for $50 \mathrm{ps}$ in a total of 7 stages. By heating gradually in stages, the possible crashes will be reduced by allowing it to equilibrate at each temperature. The heated systems were then subjected to molecular dynamics simulations for $1 \mathrm{~ns}$ each at $325 \mathrm{~K}$ in a total of 10 stages. In all simulations, the temperature was kept constant at $325 \mathrm{~K}$. The simulations were performed using the PMEMD module with GPU system which utilizes the particle mesh Ewald method [12]. SHAKE algorithm was used to constrain bond lengths involving hydrogen [13].

The mutation of p.Gly35Asp in type IX collagen alpha 3 has a Pro-Pro-Gly consecutive residue pattern. The collagen model structure was generated with a template of $2 \mathrm{v} 53$ PDB (protein database bank). After the MD simulation, the wild type and mutant collagen structures were visually inspected. The wild type structure formed hydrogen bonds with its neighboring strand while the mutant was aggregated by themselves (Figure 3A to D). In addition, the distances between the mutation site (C-alpha) of the wild type and the mutant collagen are almost twice (Figure $3 \mathrm{E}$ and F). The calculated energy state of the mutant is much lower, causing a self aggregation (Figure 3G). Considering the major structural change caused by the collagen mutant, a malfunction of the type IX collagen is expected.

\section{Conclusions}

The clinical and radiographic phenotypes of MED vary heterogenously according to the genetic mutation. COMP and MATN3 mutations show marked abnormalities in hip and knee joints. Patients with the COMP mutation have characteristics of marked alterations of hip joints leading to severe osteoarthritis in early childhood [14]. The MATN3 mutation has dysplastic epiphysis of the proximal and distal femur [15]. Clinical features of autosomal recessive MED associated with DTDST mutations include scoliosis and abnormal findings such as clubfoot, cleft palate, cystic ear swelling, and clinodactyly are present at birth in approximately $50 \%$ of individuals [16]. COL9-MED is generally the mildest form of MED and is characterized by joint pain in the hips and/or knees and stiffness presenting in the first decade of life, while radiographic abnormalities are primarily restricted to the knees with relative sparing of the hips [4]. Epiphyseal flattening and irregularity of affected joint are evident. MED-related myopathy has been reported in some families with COL9A3, COMP, and COL9A2 mutations [17,18].

We presently describe an autosomal dominant MED family with novel COL9A3 mutation. The proband and his father presented with mild knee joint pain. The height of the proband was in the 20th percentile but his father's height was average for Korean males. Radiographs of the proband showed epiphyseal changes of MED. The knee joints were most severely affected but other joints revealed minor involvement including ankle, foot, and wrist. Sparing of hip joints, no history of clubfoot or other deformities at birth, and the pattern of inheritance distinguished their conditions from the other MED caused by COMP, MATN3, and DTDST mutations. Therefore, we analyzed the genes associated with collagen type IX on the bases of 
Table 1 PCR primer sequences for COL9A1, COL9A2, COL9A3, COMP, and MATN3

\begin{tabular}{|c|c|c|c|}
\hline \multicolumn{4}{|c|}{ Primer sequences } \\
\hline \multicolumn{2}{|c|}{ COL9A1 gene } & \multirow{2}{*}{$\frac{\operatorname{Tm}\left({ }^{\circ} \mathrm{C}\right)}{56}$} & \multirow{2}{*}{$\begin{array}{c}\begin{array}{c}\text { Product } \\
\text { size (bp) }\end{array} \\
824\end{array}$} \\
\hline Exon 8-10 & F - CCGATGTGCTCCACTAACCT & & \\
\hline & R - GGCCAAGTTTAGAGCCACAG & & \\
\hline \multicolumn{4}{|l|}{ COL9A2 gene } \\
\hline \multirow[t]{2}{*}{ Exon 2} & F - CAGCTTCCTGCACTGTCTGA & 56 & 241 \\
\hline & R - GACGAGGGGCACTACATCTC & & \\
\hline \multirow[t]{2}{*}{ Exon 3-4 } & F - TGAGCCGTAGTGTGCTGTCT & 56 & 278 \\
\hline & R - CTGGAGGTCAATTGGCAGAG & & \\
\hline \multicolumn{4}{|l|}{ COL9A3 gene } \\
\hline \multirow[t]{2}{*}{ Exon 2} & F - TTGGGTCTCACCGAGGA & 56 & 293 \\
\hline & R - GCCTGGTITTCTCTCCATCA & & \\
\hline \multirow[t]{2}{*}{ Exon 3} & F - CTTGAGGGACCCCTGATTTT & 56 & 152 \\
\hline & R - TGTTCTGAGTTCCCCCTITC & & \\
\hline \multirow[t]{2}{*}{ Exon 4} & F - GCATTTGCTTCATTGCTGA & 56 & 222 \\
\hline & R - AATTAGGGCCGGACTCCTC & & \\
\hline \multicolumn{4}{|l|}{ COMP gene } \\
\hline \multirow[t]{2}{*}{ Exon 8-9 } & F - TTGAGGCGGGGTTGGGTG & 64 & 413 \\
\hline & R - ACCGTGCCGAGCCGTAGAT & & \\
\hline \multirow[t]{2}{*}{ Exon 10} & F - AGGAGTGTGACCTTTGCCTTCT & 64 & 334 \\
\hline & R - CTAGTCCAGCTTACCCCATCC & & \\
\hline \multirow[t]{2}{*}{ Exon 11-12 } & F - GAAGTCATTCTGGCCTGGTC & 64 & 518 \\
\hline & R - AGCGTTTTGTCAAAGGCTACC & & \\
\hline \multirow[t]{2}{*}{ Exon 13} & F - CGGGTAGCCTITGACAAAACG & 62 & 331 \\
\hline & R - GCCCGCCCACCGTAGAC & & \\
\hline \multirow[t]{2}{*}{ Exon 14-15 } & F - GGCGGGCCCTGACTTTAG & 64 & 546 \\
\hline & R - ATAACCCCGCCCCTCTGT & & \\
\hline \multirow[t]{2}{*}{ Exon 16} & F - GTTCTGGGTGCCAGGTTC & 64 & 335 \\
\hline & R - AAGGGTITACGGAGGGTCAT & & \\
\hline \multirow[t]{2}{*}{ Exon 17} & F - TGCTCCCAACTGTCTCTCCA & 64 & 312 \\
\hline & R - ACCTGGGCCTGTGTGTCC & & \\
\hline \multirow[t]{2}{*}{ Exon 18-19 } & F - TCTGAGAGGGAAGGGTCTGG & 64 & 443 \\
\hline & R - CCCTTCTCACTTCCCCCTCA & & \\
\hline \multicolumn{4}{|l|}{ MATN3 gene } \\
\hline \multirow[t]{2}{*}{ Exon 3} & F - AAAGGAGCCCAGAGAGCAAT & 59 & 290 \\
\hline & R - CAGTCCAAAACCTGGAGCAT & & \\
\hline
\end{tabular}

clinical and radiographic phenotypes. It is interesting that the COL9A3 mutation detected in the proband and his father is a missense mutation. Previously reported COL9A3 mutations were splice site acceptor or donor mutations in intron 2 or 3 , that invariably resulted in skipping of exon 3 of COL9A3 [17,19] with deletion of 12 amino acids in the COL3 domain of COL9A3. The c.104G> A substitution in exon 2 of COL9A3, resulting in p.Gly35Asp, affects the COL3 domain of COL9A3, and results in a substitution of Gly to Asp in the third position of "Pro-Pro-Gly" consecutive sequence. The COL3 domain is consistently affected in COL9A2 and COL9A3 mutations associated with MED, thus emphasizing the role of the COL3 domain in the pathogenesis of MED. Although, the novel mutation does not result in a splicing defect of the COL3 domain, it affects an important amino acid in a conserved "Pro-Pro-Gly" repeat sequence of the highly conserved COL3 domain.

Another point of interest is that the proband had been treated for seizure disorder. A study characterizing a microdeletion of 20q13.33, a cytogenetic locus in which COL9A3 is located, demonstrated that this abnormality is associated with several clinical features including mental retardation, developmental delay, speech and language deficits, behavior problems and seizures. In the study, two of six patients had deletions that encompassed COL9A3 and one had seizure disorder, but they lacked in clinical features reminiscent of MED. The association between seizure and COL9A3 is not clear, but the possibility should be considered. In addition, the authors remarked that the suggested deletion of COL9A3 did not cause the MED symptom [20]. However, we have a reservation with this opinion, because symptoms of MED caused by COL9A3 mutations are mild and the patients with microdeletion at 20q13.33 had combined abnormalities in morphology and psychomotor and behavioral development. It should be defined after careful clinical and radiographic assessment.

Type IX collagen is the structural component of hyaline cartilage and vitreous of the eye, and has been observed in various tissues including the notochord, inner ear, heart, brain, and skin in mice [21]. Collagen IX is a hetero-trimeric molecule that consists of three $\alpha$ chains, $\alpha 1(\mathrm{IX}), \alpha 2(\mathrm{IX})$, and $\alpha 3(\mathrm{IX}))$ in a 1:1:1 ratio. And the sizes of the domains and amino acid sequences of the $\alpha 1$ (IX), $\alpha 2(\mathrm{IX})$, and $\alpha 3$ (IX) chains are highly conserved. Also the reported mutations in COL9A2 and COL9A3 show a remarkable consistency in affecting the COL3 domain [22], and clearly indicates that the COL3 domain is a functionally important component of type IX collagen in vivo. MD simulation of heterotrimer collagen IX revealed that the wild type structure formed hydrogen bonds with its neighboring strand, while the mutant showed self-aggregation. In addition, the distances between the mutation site ( $\mathrm{C}$-alpha) of wild type and mutant collagen are almost twice and the calculated energy state of the mutant is much lower, causing a selfaggregation. Our results reveal that the substitution of Gly to Asp produces a large local disruption to the COL3 domain of COL9A3, enough to lead to a malfunction of the type IX collagen. 

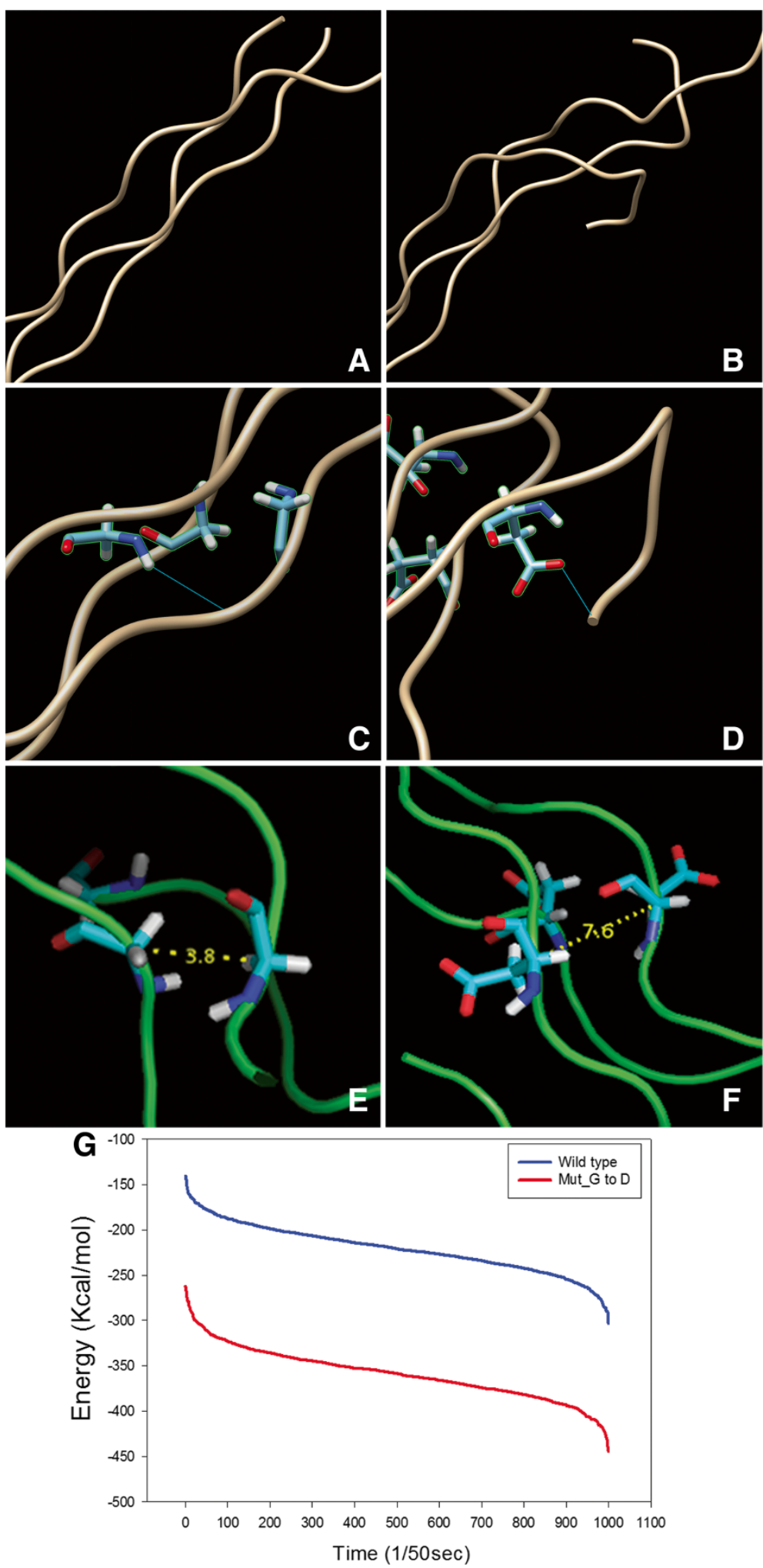

Figure 3 MD simulation of wild type and mutant model structure of trimer collagen. Wild type model structure (A), twisted form of the mutant (B), collagen-collagen interaction by a hydrogen bonding in wild type (C), the substitution of glycine to aspartic acid causing an interaction with the self strand (D), C-alpha distance between residues in wild type and mutant trimer (E and F), respectively, and energy plot of wild type and mutant collagen by Amber-GPU (G). 
Lastly, the phenotypic difference within family members was also observed but neither of the family members showed neuromuscular manifestations, a feature that has been associated in a previous MED patient with COL9A3 mutation [17]. It remains to be determined whether both inter- and intra-familial phenotypic diversity are common in type IX collagen mutations. As well, the relationship between MED and other neuromuscular manifestations must be clarified.

\section{Consent}

Written informed consent was obtained from the parents of the child for publication of this Case report and any accompanying images. A copy of the written consent is available for review by the Editor of this journal.

\section{Competing interests}

The authors declare that they have no competing interests.

\section{Authors' contributions}

$\mathrm{CJ}$ and JYL diagnosed the MED and clinically examined the family members. $J \mathrm{~K}, \mathrm{HC}$ and $\mathrm{HP}$ helped with the genetic analyses and interpretation. $\mathrm{CJ}$ and OHK provided scientific support and valuable advice. PK, YKL and JJ performed MD simulation studies. MK reviewed the literature and wrote the manuscript. All authors have read and approved the final manuscript.

\section{Acknowledgements}

We are grateful to the patient and parents assisting us in carrying out this study and compiling this report. The authors wish to acknowledge the financial support of the Catholic Medical Center Research Foundation made in the program year of 2012

\section{Author details}

'Department of Orthopaedic Surgery, Bucheon St. Mary's Hospital, The Catholic University of Korea, Seoul, Korea. ${ }^{2}$ Catholic Genetic Laboratory Center, Seoul St. Mary' Hospital, The Catholic University of Korea, Seoul, Korea. ${ }^{3}$ Department of Laboratory Medicine, The Catholic University of Korea, Seoul, Korea. ${ }^{4}$ Department of Radiology, Ajou University Hospital, Suwon, Korea. ${ }^{5}$ Syntekabio Inc, Seoul, Korea.

Received: 13 May 2014 Accepted: 23 October 2014

Published: 8 November 2014

\section{References}

1. Unger S, Hecht JT: Pseudoachondroplasia and multiple epiphyseal dysplasia: new etiologic developments. Am J Med Genet 2001, 106:244-250

2. Superti-Furga LN A, Riebel T, Eich G, Steinmann B, Spranger J, Kunze J: Recessively inherited multiple epiphyseal dysplasia with normal stature, club foot, and double layered patella caused by a DTDST mutation. J Med Genet 1999, 36:621-624.

3. Briggs MD, Chapman KL: Pseudoachondroplasia and multiple epiphyseal dysplasia: mutation review, molecular interactions, and genotype to phenotype correlations. Hum Mutat 2002, 19:465-478.

4. Jackson GC, Mittaz-Crettol L, Taylor JA, Mortier GR, Spranger J, Zabel B, Le Merrer M, Cormier-Daire V, Hall CM, Offiah A, Wright MJ, Savarirayan R, Nishimura G, Ramsden SC, Elles R, Bonafe L, Superti-Furga A, Unger S, Zankl A, Briggs MD: Pseudoachondroplasia and multiple epiphyseal dysplasia: a 7-year comprehensive analysis of the known disease genes identify novel and recurrent mutations and provides an accurate assessment of their relative contribution. Hum Mutat 2012, 33:144-157.

5. Itoh T, Shirahama S, Nakashima E, Maeda K, Haga N, Kitoh H, Kosaki R, Ohashi H, Nishimura G, Ikegawa S: Comprehensive screening of multiple epiphyseal dysplasia mutations in Japanese population. Am J Med Genet A 2006, 140A:1280-1284.

6. Kim OH, Park H, Seong MW, Cho TJ, Nishimura G, Superti-Furga A, Unger S, Ikegawa S, Choi IH, Song HR, Kim HW, Yoo WJ, Shim JS, Chung CY, Oh CW, Jeong C, Song KS, Seo SG, Cho SI, Yeo IK, Kim SY, Park S, Park SS, Kim HW,
Yoo WJ, Shim JS, Chung CY, Oh CW, Jeong C, Song KS, et al: Revisit of multiple epiphyseal dysplasia: ethnic difference in genotypes and comparison of radiographic features linked to the COMP and MATN3 genes. Am J Med Genet A 2011, 155A:2669-2680.

7. Ramensky V, Bork P, Sunyaev S: Human non-synonymous SNPs: server and survey. Nucleic Acids Res 2002, 30:3894-3900.

8. $\mathrm{Ng} \mathrm{PC}$, Henikoff S: Predicting deleterious amino acid substitutions. Genome Res 2001, 11:863-874.

9. Case DA, Cheatham TE 3rd, Darden T, Gohlke H, Luo R, Merz KM Jr, Onufriev A, Simmerling C, Wang B, Woods RJ: The Amber biomolecular simulation programs. J Comput Chem 2005, 26:1668-1688.

10. Hawkins DG, Cramer JC, Truhlar GD: Parametrized models of aqueous free energies of solvation based on pairwise descreening of solute atomic charges from a dielectric medium. J Phys Chem 1996, 100:19824-19839.

11. Izaguirre JA, Catarello DP, Wozniak JM, Skeel RD: Langevin stabilization of molecular dynamics. J Chem Phys 2001, 114:2090-2098.

12. Gotz AW, Williamson MJ, Xu D, Poole D, Le Grand S, Walker RC: Routine microsecond molecular dynamics simulations with AMBER on GPUs. 1. Generalized born. J Chem Theory Comput 2012, 8:1542-1555.

13. Ryckaert J-P, Ciccotti G, Berendsen HJC: Numerical integration of the cartesian equations of motion of a system with constraints: molecular dynamics of n-alkanes. J Comput Phys 1977, 23:327-341.

14. Briggs MD, Hoffman SM, King LM, Olsen AS, Mohrenweiser H, Leroy JG, Mortier GR, Rimoin DL, Lachman RS, Gaines ES, Cekleniak JA, Knowlton RG, Cohn DH: Pseudoachondroplasia and multiple epiphyseal dysplasia due to mutations in the cartilage oligomeric matrix protein gene. Nat Genet 1995, 10:330-336.

15. Chapman KL, Mortier GR, Chapman K, Loughlin J, Grant ME, Briggs MD: Mutations in the region encoding the von Willebrand factor $A$ domain of matrilin-3 are associated with multiple epiphyseal dysplasia. Nat Genet 2001, 28:393-396.

16. Ballhausen $D$, Bonafé L, Terhal $P$, Unger SL, Bellus $G$, Classen M, Hamel BC, Spranger J, Zabel B, Cohn DH, Cole WG, Hecht JT, Superti-Furga A, Cole WG, Hecht JT, Superti-Furga A: Recessive multiple epiphyseal dysplasia (rMED): phenotype delineation in eighteen homozygotes for DTDST mutatio R279W. J Med Genet 2003, 40:65-67.

17. Bonnemann CG, Cox GF, Shapiro F, Wu JJ, Feener CA, Thompson TG, Anthony DC, Eyre DR, Darras BT, Kunkel LM: A mutation in the alpha 3 chain of type IX collagen causes autosomal dominant multiple epiphyseal dysplasia with mild myopathy. Proc Natl Acad Sci U S A 2000, 97:1212-1217.

18. Jackson GC, Marcus-Soekarman D, Stolte-Dijkstra I, Verrips A, Taylor JA, Briggs MD: Type IX collagen gene mutations can result in multiple epiphyseal dysplasia that is associated with osteochondritis dissecans and a mild myopathy. Am J Med Genet A 2010, 152A:863-869.

19. Nakashima E, Kitoh H, Maeda K, Haga N, Kosaki R, Mabuchi A, Nishimura G, Ohashi $\mathrm{H}$, Ikegawa S: Novel COL9A3 mutation in a family with multiple epiphyseal dysplasia. Am J Med Genet A 2005, 132A:181-184.

20. Traylor RN, Bruno DL, Burgess T, Wildin R, Spencer A, Ganesamoorthy D, Amor DJ, Hunter M, Caplan M, Rosenfeld JA, Theisen A, Torchia BS, Shaffer $L G$, Ballif BC, Slater HR: A genotype-first approach for the molecular and clinical characterization of uncommon de novo microdeletion of 20q13.33. Plos One 2010, 5:e12462.

21. Nishimura I, Muragaki $Y$, Hayashi M, Ninomiya Y, Olsen BR: Tissue-specific expression of type IX collagen. Ann N Y Acad Sci 1990, 580:112-119.

22. Muragaki Y, Mariman EC, van Beersum SE, Perala M, van Mourik JB, Warman $\mathrm{ML}$, Olsen BR, Hamel BC: A mutation in the gene encoding the alpha 2 chain of the fibril-associated collagen IX, COL9A2, causes multiple epiphyseal dysplasia (EDM2). Nat Genet 1996, 12:103-105.

doi:10.1186/1471-2474-15-371

Cite this article as: Jeong et al:: Novel COL9A3 mutation in a family diagnosed with multiple epiphyseal dysplasia: a case report. BMC Musculoskeletal Disorders 2014 15:371. 